\title{
Ambulatory Treatment for Multidrug-Resistant Tuberculosis in Rural Haiti: A Mixed-Methods Study of Social and Economic Factors Affecting Timely Diagnosis and Treatment Success
}

\author{
Jude Beauchamp ${ }^{1,2}$, Carole D. Mitnick ${ }^{3}$, Hannah Gilbert ${ }^{3}$, Fernet Leandre ${ }^{1}$, Yoldine Talina Jean Noel ${ }^{1}$, \\ Walkens Desir ${ }^{1}$, Christina Lively ${ }^{2} \&$ Joia Mukherjee ${ }^{2,3}$ \\ ${ }^{1}$ Zanmi Lasante, Cange, Haiti \\ ${ }^{2}$ Program for Master of Medical Science in Global Health Delivery, Department of Global Health and Social \\ Medicine, Harvard Medical School, Boston, USA \\ ${ }^{3}$ Department of Global Health and Social Medicine, Harvard Medical School, Boston, USA \\ Correspondence: Jude Beauchamp, Partners In Health, 800 Boylston Street Suite 300, Boston, MA 02199, USA. \\ Tel: 1-561-475-8992. E-mail: juddbeauchamp@gmail.com
}

Received: April 19, 2021 Accepted: May 24, 2021 Online Published: June 16, 2021

doi:10.5539/gjhs.v13n7p99 URL: https://doi.org/10.5539/gjhs.v13n7p99

\begin{abstract}
Background and objectives: Patients with MDR-TB in remote areas of Haiti face barriers that delay their ability to initiate and complete treatment. These barriers must be addressed to ensure successful decentralization of MDR-TB treatment. We conducted a mixed-methods study at two MDR-TB facilities in rural Haiti to identify factors that impact MDR-TB treatment initiation and documented treatment response after discharge from hospital.

Methods: We enrolled patients who started MDR-TB treatment at two Zanmi Lasante MDR-TB facilities in Haiti between October 2015 and March 2016. We conducted interviews with patients at discharge to assess factors creating delay in treatment initiation; performed a longitudinal quantitative assessment of patient wellbeing at discharge and for four months after; and conducted a second interview with the same patients and family caregivers to identify obstacles to transition from hospital-based care to ambulatory treatment. Qualitative data were coded into six final conceptual categories representing patient experiences with TB treatment and care. We then explored how the patient experience during transition might affect the score obtained in the quantitative assessment.
\end{abstract}

Results: We enrolled 17 MDR-TB patients and 16 family members. Four patients were identified as having poor clinical response after discharge, characterized by low BMI, low hemoglobin, compromised activities of daily life, food insecurity, depression and other treatment side effects. Patients with poor response also experienced limited social support and difficult living conditions.

Factors contributing to delayed treatment initiation were present at discharge and may compromise response. These include financial (lost wages, costly transportation) and logistical barriers to reaching highly centralized treatment facilities. The results highlight the barriers that MDR-TB patients in rural Haiti face to find and successfully complete lifesaving treatment.

Conclusion: Properly implemented decentralized care will help overcome geographical barriers. Strong nutritional, social and financial support are essential to support appropriate treatment for the disease.

Keywords: drug-resistant tuberculosis, treatment initiation delay, treatment completion barrier, mixed-methods

\section{Abbreviations}

DR-TB: Drug-resistant Tuberculosis; MDR-TB: Multidrug-resistant TB; RR-TB: Rifampin-resistant TB; RHEZ: Rifampin/Isoniazid/Ethambutol/Pyrazinamide, referring to treatment for sensitive TB; DST: Drug susceptibility testing; ZLDSI: Zanmi Lasante Depression Symptoms Inventory; ADL: Activity of daily living; ECOG: Eastern Cooperative Oncology Group. 


\section{Introduction}

In February 2012, Alfred Simon (Note 1), a 26 year-old peasant farmer living in a remote area of Haiti, reported several months of night sweats, coughing and weight loss at a local health center and was diagnosed with tuberculosis (TB). Alfred's general presentation-markedly weak and emaciated - indicated advanced TB. The clinic initiated TB treatment immediately. After five months of regular treatment, Alfred's condition did not improve, suggesting that he had multidrug-resistant TB (MDR-TB) or rifampin-resistant TB (RR-TB) ${ }^{2}$. The staff transferred Alfred to a tertiary MDR-TB facility, where he was placed on an MDR-TB regimen. After eight months of inpatient care, Alfred was discharged with the expectation that he would return for monthly follow-up visits. A month later, the staff noticed that Alfred did not attend his first follow-up visit. While hospital staff were coordinating a home visit with Alfred, they learned from his local health center that he had died.

Alfred responded well to MDR-TB treatment in the clinic. What interfered with his treatment at home and caused him to die?

\section{Background}

Globally, MDR-TB or RR-TB (Note 2) was detected in approximately 600000 patients with TB in 2016 (World Health Organization [WHO], 2017a). In Haiti, MDR-TB is estimated to occur in $2.9 \%$ of new cases and in $13 \%$ of patients previously treated for TB (WHO, 2017b). This compares to global estimates of MDR-TB in $4.1 \%$ of new cases and in 19\% of previously treated TB patients (WHO, 2017a). Most MDR-TB is never diagnosed or reported: only 153119 cases - approximately $26 \%$ of the estimated incidence cases of MDR-TB - were notified to the WHO in 2016 (WHO, 2017a).

An even smaller proportion of people receive appropriate treatment: 126689 in 2016, which represents only 22\% of the total new patients with the disease (WHO, 2017a). Only half (54\%) of the patients whose outcomes were reported in 2014 were successfully treated (WHO, 2017a). Although undiagnosed and untreated drug-resistant TB (DR-TB) cases are very dangerous for the community (Institute of Medicine [IOM], 2011), treatment is often considered too expensive to be cost-effective in resource-poor settings (Iseman, Cohn, \& Sbarbaro, 1993; White \& Moore-Gillon, 1998).

Treatment for MDR-TB is lengthier (20 months or more in many countries, including Haiti) and more difficult for patients to tolerate than treatment for DR-TB due to frequent drug toxicities. To manage the side effects and ensure proper daily administration of injections in the first treatment phase, many countries including Haiti have adopted an in-patient model of care (Loveday et al., 2015) which providers hospitalize patients for an extended period, then discharges patients to ambulatory treatment with regular (often monthly) follow-up visits at the hospital. The treatment is highly centralized in Haiti. Most Haitians must travel long distances to access these facilities (Figure $1)$. 


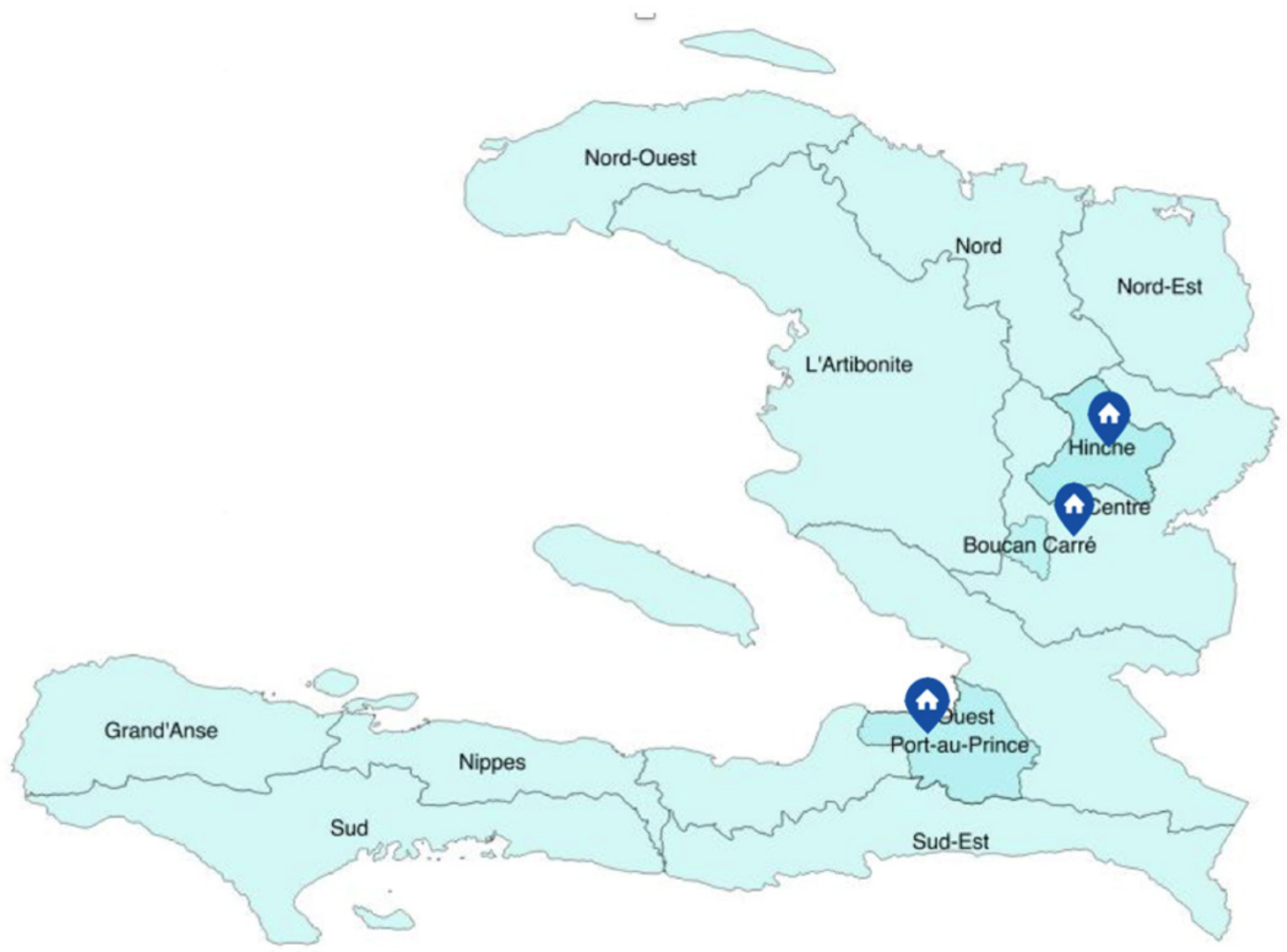

Figure 1. Map of Haiti displaying the location of the drug-resistant TB (DR-TB) treatment facilities (blue pins) of the country. (C) Jude Beauchamp

An alternative to hospital-based care is the community-based model in which MDR-TB treatment is initiated in patients' homes or in local clinics (Loveday et al., 2015). This approach has been shown to be more cost-effective than a hospital-based model (Fitzpatrick \& Floyd, 2012). It brings care providers closer to patients' homes and families (Loveday et al., 2015) and helps reduce disease transmission in the community through rapid introduction of appropriate treatment (Dharmadhikari et al., 2014).

As Alfred's case illustrates, successful ambulatory treatment depends on a patient's capacity to remain engaged with care for the full duration of the treatment course. It is therefore important to explore and understand the social forces that shape a patient's ability to access and complete treatment while living in their home communities. Evidence gathered through this line of inquiry may further inform the development of a fully ambulatory treatment program.

\section{Methods}

The research team conducted a mixed-methods study (Creswell, 2014) between June 2016 and January 2017 at Haiti's two Zanmi Lasante/Partners In Health (ZL/PIH) MDR-TB facilities: Bon Sauveur Hospital in Cange, Boucan-Carré and Sainte Thérèse Hospital in Hinche, two of the country only three MDR-TB facilities. We aimed to: 1) identify barriers and facilitators that shaped patients' experiences in seeking care and completing treatment (through interviews), and 2) explore how those factors impact patient health (through chart review and interviews) during the transition from hospital-based to ambulatory treatment.

The TB program manager informed prospective patients about the study and asked about their willingness to participate. With their permission, the TB program manager introduced patients to the local primary investigator (PI) for enrollment. The PI enrolled all eligible, adult ( $\geq 18$ years) patients with culture- and DST-confirmed MDR-TB who started treatment at the study sites between October 2015 and March 2016. Patients with cognitive limitations that could impair their abilities to provide informed consent or participate in the interviews were excluded.

The study also gathered information from family caregivers charged with overseeing a patient's MDR-TB care to 
better understand their experiences of providing care to MDR-TB patients. Family caregivers were referred to the researcher by enrolled patients, and were consented prior to participation.

\subsection{Quantitative Measures}

Routinely collected clinical data (treatment side effects [SE], microbiology results, body mass index [BMI], hemoglobin $[\mathrm{Hb}]$, chest X-ray [CXR]) from each of the first four months of ambulatory treatment (transition period) were recorded in MS Excel (v2016). Investigators also performed brief, validated surveys to capture signs of depression, patients' overall condition and ability to perform activities of daily living (ADL) and degree of food insecurity (FI). Information collected was collapsed into six indicators - clinical, treatment side effects, depression, overall condition, food insecurity, and microbiology — with a score assigned to each indicator. Description of indicators and their scoring are detailed in Table 1. Higher scores indicate more signs of compromised treatment response.

\subsection{Qualitative Data Collection}

Data collection for the qualitative study included two individual, semi-structured interviews with each patient participant. All interviews were conducted by the PI in Haitian Creole, and audio-recorded with permission. Initial interviews were one hour in length, and took place in a private room at the hospital during the in-patient treatment phase. Interviews used open-ended questions to explore the following topics: (1) experiences during the care-seeking process before treatment initiation; (2) duration of care-seeking trajectory (3) treatments they had tried; (4) their TB history; and (5) barriers to seeking appropriate MDR-TB care. Follow-up interviews took place in patients' homes during the transition period-defined as the first four months of ambulatory treatment. Interviews averaged half an hour in length, and explored the following topics: (1) overall experience with ambulatory treatment; (2) barriers to completion of follow-up visits; and (3) facilitators of treatment continuation.

Ethnographic observations during the home visits, allowed the PI to learn more about patients' living conditions and observe caregiving first-hand in patients' normal setting (Atkinson, Coffey, Delamont, Lofland, \& Lofland, 2001).

\subsection{Quantitative Analysis}

Using the clinical response scoring system described in Table 1, we summed each patient-month score. We then identified patients whose response scores were in the top $25 \%$ of scores for at least 3 months. We considered these patients to have the most compromised response during transition from hospitalization and ambulatory care. We explored the qualitative data to inform how patient circumstances at transition reflect those prior to diagnosis and the possibilities for interventions to improve prospects for treatment response in at-risk patients. We used STATA version 14.2 (College Station, Texas) for the analysis.

\subsection{Qualitative Analysis}

Qualitative data analysis relied on inductive content analysis, which produced a set of key thematic concepts. A sub-set of transcripts were open-coded to produce a codebook, which was then piloted and revised. The final codebook was used to code the entire dataset of transcribed interviews. Coded data was then analyzed inductively to construct initial descriptive categories (Patton, 2014; Corbin, \& Strauss, 2008). These provisional categories were revised and elaborated into six final conceptual categories representing patient experiences with TB treatment and care that are reported in the results section below.

\subsection{Integration of Quantitative and Qualitative Results}

After the independent analysis of the quantitative and qualitative data, we explored how the patient experience during transition might affect the score obtained in the quantitative assessment (Figure 2). 


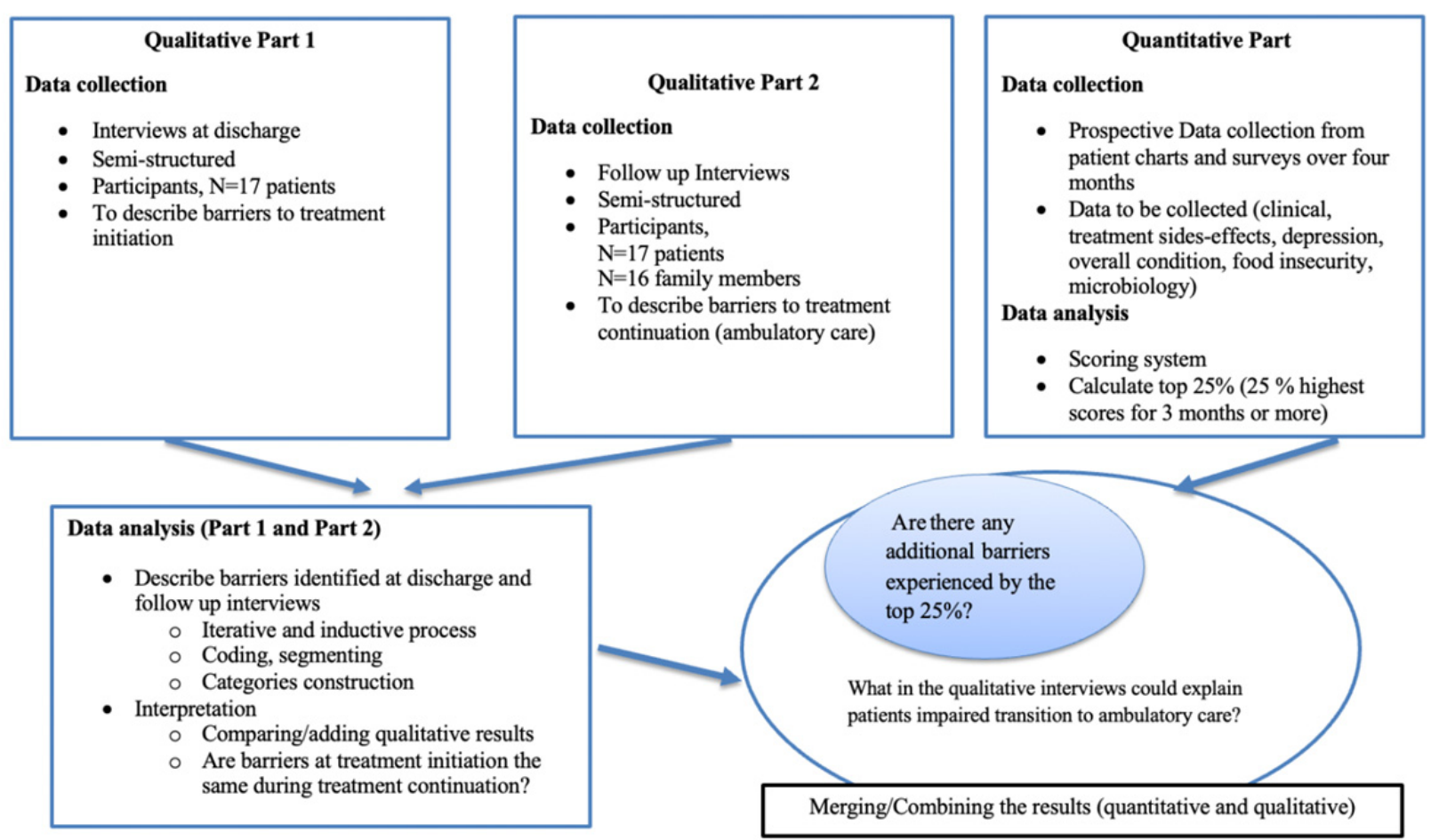

Figure 2. Study design diagram

Table 1. Components of clinical response score

\begin{tabular}{|c|c|}
\hline Indicator & Scoring \\
\hline $\begin{array}{cl}\text { Clinical } & \\
\text { - } & \text { Body Mass Index (BMI) (Note 3) } \\
\text { - } & \text { Chest X-ray (CXR) (Note 4) } \\
\text { - } & \text { Hemoglobin (Hb) (Note 5) }\end{array}$ & $\begin{array}{l}\text { No abnormal }=0 \\
\text { One abnormal }=1 \\
\text { More than one abnormal }=2\end{array}$ \\
\hline Treatment side effects (SE) & $\begin{array}{l}\text { No } \mathrm{SE}=0 \\
\text { One } \mathrm{SE}=1 \\
\text { More than one } \mathrm{SE}=2\end{array}$ \\
\hline $\begin{array}{l}\text { Depression using the validated Zanmi Lasante Depression Symptoms Inventory } \\
\text { (ZLDSI). }\end{array}$ & $\begin{array}{l}\text { Scale }<13=0 \\
\text { Scale } \geq 13=1\end{array}$ \\
\hline $\begin{array}{l}\text { Overall condition (ADL) using the Eastern Cooperative Oncology Group } \\
\text { (ECOG) performance status. }\end{array}$ & $\begin{array}{l}\text { No worsening }=0 \\
\text { Worsening }=1\end{array}$ \\
\hline Food insecurity (FI), using the validated hunger scale survey. & $\begin{array}{l}\text { No food insecurity }=0 \\
\text { Food insecurity }=1\end{array}$ \\
\hline $\begin{array}{l}\text { Microbiology } \\
\text { - } \quad \text { Culture of sputum exam } \\
\text { - } \quad \text { Drug susceptibility testing }\end{array}$ & $\begin{array}{l}\text { Negative culture }=0 \\
\text { Positive culture }=2 \\
\text { Resistance amplification (in DST) }=3 .\end{array}$ \\
\hline
\end{tabular}

\section{Results}

\subsection{Quantitative Findings}

Between June and October 2016, 17 patients began MDR-TB treatment at the two ZL/PIH MDR-TB treatment centers. All patients enrolled for MDR-TB treatment during the study period agreed and were eligible to participate in the study. Of these, $13(76.4 \%)$ were receiving treatment at Bon Sauveur Hospital and 4 (23.5\%) at 
Sainte Therèse Hospital. Roughly half (9 [52.9\%]) were female, high-school educated, and living with their parents. Mean age was 28 (range: 18 to 52) years. More than half, (11 [64.1\%]), lived more than 100 miles from the MDR-TB treatment center where they received treatment (Table 2). Eight (47.0\%), were self-employed (street vendors, peasant farmers) at time of TB diagnosis. Two (11.7\%) patients reported formal employment at the time of TB diagnosis.

Table 2. Socio-demographic characteristics of the study population

\begin{tabular}{|c|c|}
\hline \multicolumn{2}{|l|}{ Age } \\
\hline $18-30$ & $14(82.3 \%)$ \\
\hline $31-50$ & $2(11.7 \%)$ \\
\hline$>50$ & $1(5.8 \%)$ \\
\hline \multicolumn{2}{|l|}{ Sex } \\
\hline Female & $9(52.9 \%)$ \\
\hline Male & $8(47.0 \%)$ \\
\hline \multicolumn{2}{|l|}{ Education level } \\
\hline Secondary or higher & $11(64.7 \%)$ \\
\hline Primary & $4(23.5 \%)$ \\
\hline No school & $2(11.7 \%)$ \\
\hline \multicolumn{2}{|l|}{ Marital status } \\
\hline Single independent & $1(5.8 \%)$ \\
\hline Single living with parents & $9(52.9 \%)$ \\
\hline Married or living with partner & $7(41.1 \%)$ \\
\hline \multicolumn{2}{|l|}{ Distance from home to MDR-TB clinic } \\
\hline$<100$ miles & $6(35.2 \%)$ \\
\hline$\geq 100$ miles & $11(64.1 \%)$ \\
\hline \multicolumn{2}{|l|}{ Occupation } \\
\hline Employed & $2(11.7 \%)$ \\
\hline Unemployed & $2(11.7 \%)$ \\
\hline Self-employed (street vendor, peasant farmer) & $8(47.0 \%)$ \\
\hline In school & $5(29.4 \%)$ \\
\hline
\end{tabular}

\subsubsection{Quantitative Results During Transition}

The mean monthly clinical response score was 3 (range: 1-8). Scores were relatively stable over the four-month transition period (table 3 ). Four patients (table 4) consistently had scores in the top $25 \%$ for three or four months (table 5). No other patient had any monthly score in the top $25 \%$ (table 5). The signs and symptoms most commonly present among patients with higher scores included low BMI, low HB, limited ADL capacities, more SE, and positive depression screening (ZLDSI) (table 5). Patients in the top 25\% group also reported more food insecurity and a less reliable social support system. FI and SE were common in the cohort (table 5).

Table 3. Treatment response score after discharge

\begin{tabular}{lllllllll}
\hline & \multicolumn{2}{l}{ Month 1 } & \multicolumn{2}{c}{ Month 2 } & \multicolumn{2}{c}{ Month 3 } & \multicolumn{3}{c}{ Month 4 } \\
& Mean & Range & Mean & Range & Mean & Range & Mean & Range \\
\hline Score for all patients & 3.7 & 1,8 & 3.7 & 1,7 & 3.3 & 1,7 & 3.5 & 1,7 \\
Score with compromised response & 6.2 & 5,8 & 5.5 & 5,7 & 5.2 & 4,7 & 5.5 & 5,7 \\
\hline
\end{tabular}


Table 4. Baseline characteristics of patients with compromised response

\begin{tabular}{|c|c|c|c|c|}
\hline $\begin{array}{l}\text { Pseudonym (Note 1) } \\
\text { Baseline Characteristic }\end{array}$ & Kathy & Darline & Martine & Gregory \\
\hline Age & 18 уо & 22 уо & 18 yo & 25 yo \\
\hline Gender & $\mathrm{F}$ & $\mathrm{F}$ & $\mathrm{F}$ & M \\
\hline Education & High school & High school & Primary school & High school \\
\hline Domicile & North & North & North & North \\
\hline $\begin{array}{l}\text { Occupation before TB } \\
\text { disease }\end{array}$ & High school student & High school student & None & Small business owner \\
\hline Living conditions & $\begin{array}{l}\text { Live with and rely on } \\
\text { parents }\end{array}$ & $\begin{array}{l}\text { Live with and rely on } \\
\text { partner }\end{array}$ & $\begin{array}{l}\text { Live with and rely on } \\
\text { relatives }\end{array}$ & $\begin{array}{l}\text { Live alone but relies on } \\
\text { relatives }\end{array}$ \\
\hline Treatment facility & Cange & Cange & Cange & Cange \\
\hline
\end{tabular}

Table 5. Characteristics over four months for patients with compromised transition after discharge

\begin{tabular}{lllll}
\hline Pseudonym & Kathy & Darline & Martine & Gregory \\
\cline { 2 - 5 } Characteristic & Mean & Mean & Mean & Mean \\
\hline Body Mass Index (Note 6) & $16.8(16.4,17.2)$ & $21.2(20.3,22.0)$ & $16.0(15.6,16.4)$ & $18.4(17.0,19.6)$ \\
Hemoglobin (Note 6) & $12.5(10.6,14.1)$ & $12.6(12,13)$ & $11.3(11.0,11.6)$ & $11.5(11,11.8)$ \\
Side Effects (Note 6) & $1.5(1,2)$ & $2.5(2,3)$ & $1.2(1,2)$ & $2(2,2)$ \\
Depression (Note 6) & $12.5(11,15)$ & $15.2(14,16)$ & $10.5(9,12)$ & $15.7(15,16)$ \\
Activities of Daily Life (Note 6) & $0.75(0,1)$ & $1(1,1)$ & $1(1,1)$ & $1.7(1,2)$ \\
Food Insecurity (Note 6) & $1(1,1)$ & $1(1,1)$ & $1(1,1)$ & $1(1,1)$ \\
\hline
\end{tabular}

\subsection{Qualitative Findings}

Participants' pseudonyms and characteristics are provided in Table 6.

Table 6. Characteristics of patients

\begin{tabular}{llll}
\hline Age & Sex & Marital Status & Occupation \\
\hline $\mathbf{3 3}$ & M & living with partner & Street vendor \\
$\mathbf{2 3}$ & M & Single living with parents & High school and vocational student, DJ, field worker at a local NGO \\
$\mathbf{2 9}$ & F & Married & street vendor \\
$\mathbf{2 5}$ & M & Single living with parents & College student \\
$\mathbf{2 5}$ & M & Single living with parents & Vocational student \\
$\mathbf{2 7}$ & M & Single living with parents & Aide at a small business \\
$\mathbf{1 9}$ & F & Single living with parents & High school student \\
$\mathbf{2 9}$ & F & Married & Street vendor \\
$\mathbf{2 5}$ & M & Single living with parents & High school and vocational student, peasant farmer \\
$\mathbf{1 8}$ & F & Single living with parents & High school student \\
$\mathbf{5 2}$ & F & Married & Street vendor, peasant farmer \\
$\mathbf{2 9}$ & F & Single living with parents & No occupation reported \\
$\mathbf{2 2}$ & F & living with partner & High school student \\
$\mathbf{2 5}$ & M & Single independent & Small business owner \\
$\mathbf{1 8}$ & F & Single living with parents & High school student \\
$\mathbf{3 4}$ & M & Married & Peasant farmer \\
$\mathbf{2 7}$ & F & living with partner & Street vendor, peasant farmer \\
\hline & & &
\end{tabular}


The qualitative analysis revealed socio-economic, demographic and structural barriers that patients with MDR-TB in rural Haiti must overcome to find and complete appropriate treatment. The following themes emerged from the analysis.

\subsubsection{Prioritize Family Well-Being Over Self-Care}

In Haiti, a wage earner is often responsible for many dependents even if they have a very low income (Verhoogen, 1996). Some participants who were the primary wage earners explained that familial responsibility weighed so heavily, they were willing to prioritize their family's well-being over their own health. As these wage earners often engaged in hard physical labor, they reported that the strains from such work left them weak, and vulnerable to the disease:

I used to sell "recho" [handmade stove] ... I use sheet metal to make those stoves. Also, I may buy what I find and go out sell it, make a living from that... save additional money made from that and buy other things to sell in the street. It is the impact of that work that is breaking me now (Male, 33 years old, initial interview).

Upon returning home after discharge from hospital, some patients experienced conflict between prioritizing recovery and pursuing paid work. Having families economically dependent upon them, some often resumed work before they were physically ready.

You know things are not okay. Things are very difficult. I am making a lot of sacrifice, you understand. I have found something [a job] at the Red Cross. It's all we have. The body is not ready yet for that, but I am obliged to do it, I must go out make a living to help my family out (Male, 23 years old, second interview).

\subsubsection{Persevering Through Travel Difficulties}

Patients living in remote rural areas experienced difficulty obtaining transportation to attend monthly appointments. Many must take motorcycles across difficult terrain-a physically grueling and hazardous journey. One patient described her 130-mile journey for follow-up visits:

Sometimes you wake up early, to find transportation; it is a headache. I used to go out by 4 am. But, you wouldn't find a car until after the sun rises. Then you must take a motorcycle, then another motorcycle. The motorcycles drivers don't want to carry only one passenger, they want to wait for more passengers before leaving, that makes me wait a lot to find one (Female, 46 years old, first interview)

These journeys also caused financial strain. Many patients reported difficulty paying for transport to their follow-up visits. In such cases, some would borrow money for transport fares. Others, unable to pull together any transportation funds, resorted to undertaking the journey to the clinic on foot:

[I can] find food to eat, thank God. The only problem I had was sometimes I did not have money to pay for transportation to go to the clinic, then I walked (Female, 52 years old, first interview).

\subsubsection{Distance from Treatment Center Entails Choice between Work and Care}

Living far from treatment sites, patients described long journeys (often days) to report to monthly follow-up visits. Patients were forced to decide whether to fulfill requirements of their paid employment or schooling, or follow through on their ambulatory care appointments:

They put me on RHEZ [regular TB drugs] and told me it will be for 6 months. While I was taking the drugs, at my job they transferred me to a new position at another location. I told them "no" because I am under treatment...If I leave I won't be able to find the drugs. They [the company] told me I may go, they will help me have the drugs. I thought it was true. But they didn't do that for me. The distance was far...It became impossible for me to continue with the treatment... (Male, 27 years old, first interview).

Other patients elected to pursue treatment at the expense of incurring lost work time. Those who chose to pursue treatment and miss work often experienced significant collateral losses:

Finding a job is very difficult those days... when I go to appointments, I must miss 3 days of work, it is because the supervisor knows me, he helps me. But he takes the money for those missed days, I resigned myself [to pay him] because this helps me not to lose the job after all (Male, 23 years old, second interview).

\subsubsection{System-Related Delays in Diagnosis and Treatment}

In addition to barriers that limited patients' ability to reach the clinics, delays from within the health system also constrained patients' access to appropriate care. The first system-related delay is characterized by a failure to properly diagnosis TB. Patients explained that initial contacts with the health system often resulted in a misdiagnosis - with symptoms being glossed as a simple cough or fever. Often participants reported that 
tuberculosis was suspected only after multiple treatment attempts for persistent cough:

Starting in June, it became worse because I was coughing more, couldn't eat. I went to the hospital.... All the times I went to the center, they never did smear exam for me ... they always gave me cough syrup. It was at the last place that I went in Milot [in the north department], the nurse told me she will do smear exam for me because I was coughing so much (Female, 22 years old, first interview).

Another delay commonly reported by patients was the failure to diagnose MDR-TB. In these cases, patients were provided treatment for drug-sensitive TB, when, in fact, they probably had MDR-TB:

At the clinic, they put me on TB treatment for 6 months. They told me if I take the drugs well, I would be cured. But the treatment did not work, they placed me on another 8-month treatment regimen, for a total of 14 months of treatment, but that did not work (Female, 27 years old, first interview).

The final delay noted by participants was linked to a breakdown in the referral process, when patients with confirmed cases of MDR-TB were unable to reach appropriate treatment centers. Referring clinics sometimes faced logistical problems (broken ambulance, lack of fuel) that resulted in lengthy delays for patient transfers:

It took them a while to take me to Cange. There was transportation problem... I was supposed to be sent in November. I finally got there in February (Male, 25 years old, first interview).

\subsubsection{Inappropriate Diagnoses Pushed Patients to Non-Biomedical Healing Paths}

Several patients explained that inappropriate treatment at referring clinics eroded trust in the effectiveness of biomedical solutions, after several unsuccessful attempts to obtain effective care for their disease, some patients sought alternate healing paths outside of formal health systems:

You know I went to several hospitals in Port-au-Prince...nothing worked. After that, I called my mom, she asked me to join her [in the countryside]....she wanted to find treatment for her son. They took me to the hougan, we spent a lot of money. It was my cousin who has a friend who is a nurse, who encouraged us to come to the [hospital]. (Male, 25 years old, first interview).

The protracted cycles of ineffective care seeking resulted in significant losses for patients and their families including loss of money, and loss of time:

I went to the hospital... They told me I had malaria and gave me drugs for that... I went to another clinic, they told me I had typhoid, gave me drugs, nothing worked. A friend advised me to go to another hospital... I tried another hospital in town, nothing worked. My mom started to think about witchcraft, she took me to a hougan who had us buy several things (simagri). Nothing worked, we paid the hougan a lot ... he made me waste a lot of time (Male, 23 years old, second interview).

\subsubsection{TB and Compounded Economic Vulnerability}

The physical limitations imposed by the disease curtailed patients' capacity to contribute to their families' well-being, whether through labor within or outside of the home. Patients and families explained that when the patient with MDR-TB was the primary wage earner, the loss of income had devastating effects on family finances:

Because of his disease, he's on treatment, he can't work anymore, that makes me the only one trying to make a living for the family... And he was the head of the family, he used to do whatever he could for his family. Now, he is bedridden, that makes thing bad for us, we are doing nothing. Everything falls flat for us (Wife of a 37-years old patient, second interview).

The debts incurred during long care-seeking journeys are poised to have lasting effects on the families of MDR-TB patients. Families who have spent all of their available resources on their loved one's care reported resorting to loans in order to obtain care:

At one point, we went to spiritual healers with him, things were exaggerated for us, we were totally depleted. "Ame Seles" [spiritual healers group] took their part; every time they had to work for us we had to pay USD 55, besides buying a lot of things they asked for. Then we went to another group... We now had to take loan at "Fonkoze" [a local cooperative] to pay spiritual healers to treat him (Sister of a 23-years old male patient, second interview).

\subsection{Integrating Qualitative and Quantitative Results}

The interviews carried out during hospitalization and after discharge suggest that the factors present for the patients and their families at treatment initiation persist during treatment continuation and after discharge. These conditions may be reflected in changes in treatment response after discharge, wrought by the persistence of factors in the patients' environment. Low clinical response score (the top 25\%) was driven by low BMI, food insecurity, 
impaired ADL capacities, risk of depression. Interviews reveal an important lack of socio-economic and nutritional support as the following two patients reported:

Things are very hard for us now. Everything we had ... we sold, to cover expenses related to treatment tried. ... we have nothing left to spend and I am asking if it is possible to have some kind of support, like food, Doc. I should not be ashamed to ask for that (Male, 36 years old, first interview).

Life is very hard, "se tire wap tire" like you are pulling a heavy load. To go to appointments you couldn't find nothing, and things were hard for the family as well. We used to wake up and have nothing to eat for the day (Female, 18 years old, first interview).

In our field work and observation, we also noticed that three of the four patients (the top 25\%) were among those who had the worst living conditions.

\section{Discussion}

This study assesses factors that contribute to delays to appropriate treatment for patients with MDR- TB in rural Haiti and explores the impact of these factors on patient well-being during ambulatory care after hospital discharge. To our knowledge, this study is the first to explore the transition from in-patient to outpatient care. We employed a mixed-methods approach, using quantitative data to characterize patients' clinical response after discharge and qualitative interviews to record lived experiences, including challenges patients faced during transition.

Previous research identified risk factors for default from treatment. Some studies report associations between treatment default and patients' socio-demographic characteristics, including being male (Jha et al., 2010) and being the financial head of household (Rutherford, Hill, Maharani, Sampurno, \& Ruslami, 2013). Other factors included geographic distance to clinics (Rutherford et al., 2013) and poor satisfaction with providers and services (Rutherford et al., 2013). Here, we focus on signs at transition, arguably one of the most vulnerable times for patients receiving a combination of in- and out-patient treatment for TB. This study can inform the design of decentralized care, assuring that patients who receive all or part of their treatment in their community have the support necessary to successfully complete treatment.

The quantitative results reported here reveal variability in response across patients during the transition, suggesting the possibility of identifying patients who require additional support: four patients had scores indicating compromised response over the entire four-month transition period. Those with high clinical response scores were more likely to have difficult socio-economic conditions. Notably, none of these patients had demonstrable improvement in the four months after discharge. While this may be due to the relatively short period of observation (a possibility that is supported by the observation that there was also not detectable deterioration using our scale) the persistent score may also be due to the patients' continued adverse socioeconomic conditions. This is not surprising considering a large body of literature that highlights the direct and indirect ways that socioeconomic conditions affect the disease and illness experience. In fact, many studies from diverse settings - including high-, middle- and low-income countries-have demonstrated that socio-economic conditions are an important driver of TB treatment outcome (Djibuti, Mirvelashvili, Makharashvili, \& Magee, 2014). Favorable treatment outcomes were associated with high levels of education and high household income levels (Djibuti et al., 2014). Adverse treatment outcomes were associated with unemployment, homelessness (Diel \& Niemann, 2013), and lower levels of education (fewer than 6 years of school) (García-García et al., 2002).

Delay to appropriate tuberculosis treatment is often attributed to the patient's lack of awareness of the disease Li et al., 2013). While promoting disease awareness in the general population is important, our qualitative data indicate that treatment delays were not the result of patients failing to notice their "worsening" cough. Instead, these delays occurred despite escalation of care-seeking, because of structural limitations that impacted patients' abilities to seek and find treatment for their worsening symptoms. Those limiting factors were felt in the patient's most immediate environment-their home-where economic pressure forced patients to make decisions that prioritized their family wellbeing at the expense of their own.

Patients' spiritual beliefs and presumed lack of knowledge about the disease have been blamed for default from TB treatment; these have been cited as the reason patients turn to non-biomedical care (Barker et al., 2006). In this study, the data demonstrate that dissatisfaction and frustration with inappropriate care delivered in formal biomedical settings ultimately forced patients to seek alternative forms of treatment. Inappropriate diagnosis and treatment — not lack of education and knowledge — drove patients to pursue non-biomedical care available within their own communities (Banerjee, Harries, Nyirenda, \& Salaniponi, 2000). Our data challenge the notion that the burden of disease rests on patients and their presumed, static "cultural" beliefs and practices. Such "immodest claims of causality" are pervasive in analyses of infectious diseases in resource-poor settings (Farmer, 1997). Our 
findings suggest that focusing on patients' education and awareness is short-sighted, and does not consider the drivers that limit appropriate diagnosis and treatment of TB in this context. Research in other resource-limited settings has shown that investment in strengthening diagnostic and care capacity can minimize some of the care-seeking delays reported here (Cox et al., 2015).

Qualitative findings in our study indicate that when personal or other health needs competed with TB treatment, many patients prioritized their TB treatment regimen. In other contexts outside of Haiti, employment has been associated with non-adherence to TB treatment (Jha et al., 2010; Rutherford et al., 2013), because patients had a difficult time leaving work to pursue follow-up visits. In contrast, our study documents that many patients prioritized their treatment above their work responsibilities, accumulating missed work and study time.

Prioritizing TB treatment often came with significant financial loss. In fact, income loss due to TB disease and/or treatment has been associated with an increase in financial risk for patients. A recent systematic review of total TB cost demonstrated that income loss constituted between 16 and 94\% (unweighted average 60\%) of TB total cost (Kemp, Mann, Simwaka, Salaniponi, \& Squire, 2007). These percentages could be higher for those living in poverty as total treatment costs represent an even higher percentage of household income (Kemp et al., 2007). As the present study also suggests, income loss is significantly higher for DR-TB patients because they face a longer period of unemployment due to longer care-seeking process and a lengthier treatment regimen (Rouzier, Oxlade, Verduga, Gresely, \& Menzies, 2010). These findings highlight the importance of establishing mechanisms to protect patients' earning potential. In other contexts, financial support has been effective in improving TB treatment outcomes Wingfield et al., 2016). Anti-discrimination employment laws have been introduced in other settings to offer important protection for formal wage earners (Orsini-Duse, M/XDR-TB AND THE LAW: South African Context [PowerPoint slides]. $\quad$ Retrieved from https://www.who.int/tb/events/archive/icmeeting_oct07/03_duse.pdf) while they seek care and complete treatment.

Similar to other studies, we find that distance (Feachem, Kjellstrom, Murray, Over, \& Phillips, 1993), difficulty to travel (Mishra, Hansen, Sabroe, \& Kafle, 2005), and cost of transportation (Herrero, Ramos, \& Arrossi, 2015) constitute barriers to appropriate treatment for diagnosed MDR-TB. In addition, our data point to challenges in referring patients from TB clinics to the drug-resistant TB centers. The current study findings suggest that decentralization of treatment is key to alleviating the distance burden patients face. Other studies have demonstrated that decentralization of care in addition to access to rapid diagnosis decreased delay to treatment initiation, potentially reducing transmission risk (Berhanu et al., 2016).

This study found further delay is attributable to a lack of available diagnostic materials. Patients described waiting for long periods to receive TB test results before initiating treatment. Other patients' descriptions of their care-seeking trajectories indicate that they were initially treated for TB when they likely had primary drug-resistant TB. This points to the importance of scaling-up access to new, simple testing technologies that could critically identify TB and primary drug-resistant TB in high-burden settings such as remote rural Haiti. The effectiveness and feasibility of such technology has been confirmed in other resource-limited settings where MTB/RIF scale-up has improved accessibility to early diagnosis and treatment of TB and drug-resistant TB (Jacobson et al., 2013; Naidoo et al., 2014).

TB and MDR-TB represent a significant financial burden for patients and their families (Rouzier et al., 2010). While TB drugs are generally provided free of charge in Haiti, accessing such drugs requires that patients pay for transportation, travel-related expenses, and lab exams. These ancillary costs represent a significant sum for patients in our study. When considering DR-TB treatment, costs can be higher because the care-seeking process is often longer (Rouzier et al., 2010). Although we did not measure total cost in the present study, patients' care-seeking process suggests a heavy toll of the disease or treatment or their economic wellbeing. This points to the need to address the economic impact of the disease-its treatment-with income replacement at the same time we address the clinical aspects of the disease with medical care.

Even though MDR-TB treatment brings many long-lasting side effects, which often prevent patients from returning to normal activities (Morris et al., 2013), patients in our sample reported feeling obliged to resume work before feeling physically ready. Patients' living conditions-which were driven by poverty-left them vulnerable to disease and constrained their access to treatment and their capacity to continue with the treatment. This trajectory is indicative of how poverty and difficult living conditions determine both who gets sick and who can access treatment (Farmer, 1997). This is reflective of a pattern of "structural violence" in which socio-economic forces shape risk for TB disease (living in poverty, overcrowding) and determine access to effective treatment (Farmer, 1997). Furthermore, our study demonstrates that structural inequalities shape patients' abilities to effectively 
engage with the long-term requirements of drug-resistant TB treatment. These findings may illuminate the literature on associations between poor economic conditions (or proxies) and treatment default and may highlight the importance of alternatives (such as cash transfer, stipend allowance) to help patients to continue with the treatment upon return to home after an extended hospitalization (Djibuti et al., 2014).

\section{Limitations}

This study has several limitations. The scoring system has not been validated; some sub-indicators were validated, such as food insecurity scale and depression scale. The scoring system was used only to identify study participants whose experience would be highlighted in the qualitative part of the study. It was not used for interventions or other classification so validation was not a high priority. Moreover, the cohort was too small to be used for both validation and application of the scoring system. Discriminatory power of the scale may be compromised: it did not capture changes (worsening or improvement) in patients' clinical evolution, probably because of our small sample size and short follow-up period (only four months). In this study all missing values were coded to 0 . In the sensitivity analysis (conducted by replacing all missing data with the most abnormal score, 2 for culture, 1 for all the other sub-indicators), the mean score increased. More patients had scores in the highest $25 \%$ at least once, while two (instead of four) remained in the top percentile for 3 months or more. More patients may have experienced a compromised clinical response during the transition period. Nevertheless, the score provides a starting point for clinicians and programs to provide increased attention and support to patients with signs of poor response during transition.

\section{Conclusion}

Study patients' experiences provide further evidence of the impact of living conditions on patient ability to initiate and sustain appropriate DR-TB treatment. The qualitative results highlight the socio-demographic, geographical and structural barriers that patients must overcome to access and succeed with their treatment. Our data suggest three key recommendations that may reduce the impact of these structural constraints (Table 7). First, the decentralization of DR-TB treatment services, placing treatment centers closer to where patients live, would alleviate the significant transportation barrier reported by patients, their families and health facilities. Second, scaling up diagnostic tests that can rapidly detect TB and resistance across the public system, and initiating collaboration with the private sector could help reduce the mismanagement and delays to appropriate treatment identified here. Third, financial enablers need to be incorporated into the model to assure effective, timely treatment. Some strategies that may be applicable include direct cash transfers, disability grants, and the institution of social protection and anti-discrimination employment laws.

The quantitative measures suggest that it may be possible to detect a compromised clinical response with no demonstrable improvement over the four-month period after discharge. Qualitative interviews reveal that patients with higher clinical response scores had difficult socio-economic conditions. While Haiti has yet to fully implement ambulatory care, if we want to be successful with DR-TB treatment we should identify patients who are at higher risk of poor transition after discharge, and adjust the socio-economic support provided based on their need.

Table 7. Framework for improving accessibility to and outcome of MDR-TB treatment in Haiti

\begin{tabular}{lll}
\hline What needs to be done? & Why? (Based on study results) & How? (Policy to put in place) \\
\hline $\begin{array}{l}\text { Identify patients at risk } \\
\text { of poor transition after } \\
\text { discharge }\end{array}$ & $\begin{array}{l}\text { Quantitative results indicate variability } \\
\text { and worsening of treatment response } \\
\text { (Hospital-based }\end{array}$ & $\begin{array}{l}\text { Policy should identify patients with higher risk of poor } \\
\text { transition after discharge to provide additional } \\
\text { socio-economic and nutritional support based on the } \\
\text { settings). }\end{array}$
\end{tabular}

Primary wage earners, because of economic pressure to provide, must Address economic continue to work while sick or getting hardship that could sicker. delay seeking care and/or derail the treatment.
Government and NGOs should provide financial support for income replacement; cash transfer, grant to help generate income through agricultural activities or small businesses.

Advocacy is required from civil sector, and supported and National TB Program (NTP) for the adoption of anti-employment discrimination laws, social protection to shield patients from losing jobs or income because of TB disease and/or its treatment. 
Patients care-seeking trajectory suggests they received symptomatic treatments (for cough, fever) often in private clinics or were treated multiple times for drugs Improve rapid sensitive TB while they may have had appropriate diagnostic drugs resistant TB disease.

and treatment of TB Because of non-improvement from /DR-TB

inappropriate diagnosis and treatment of DR-TB, patients turned to non-biomedical care which is often more available to them (in their communities).

\begin{tabular}{|c|c|c|}
\hline $\begin{array}{l}\text { Incorporate private } \\
\text { sector (physicians) in } \\
\text { national TB strategy. }\end{array}$ & $\begin{array}{l}\text { Private sector providers represent } \\
\text { important resources patients with TB } \\
\text { consult while seeking care }{ }^{1} \text {. } \\
\text { Patients care-seeking trajectory reported } \\
\text { suggests inappropriate, symptomatic } \\
\text { treatment for TB disease. This pattern is } \\
\text { known as a cause of delay to appropriate } \\
\text { TB treatment }{ }^{2} \text {. }\end{array}$ & $\begin{array}{l}\text { Government should seek a partnership with private } \\
\text { providers in which: } \\
\text { The NTP should 1) train private providers on TB } \\
\text { treatment and promote awareness on national TB } \\
\text { guidelines. 2) Simplify access to rapid diagnostics and } \\
\text { anti-TB treatment. 3) ensure and that diagnosis and } \\
\text { treatment are free, even if accessed through private } \\
\text { sector; 4) facilitate case reporting, monitoring, and } \\
\text { contact tracing in conjunction with private providers }\end{array}$ \\
\hline \multirow{3}{*}{$\begin{array}{l}\text { Decentralization } \\
\text { DR-TB treatment }\end{array}$} & $\begin{array}{l}\text { Demographic data highlight distance } \\
\text { challenges patients must overcome to } \\
\text { reach DR-TB facilities. }\end{array}$ & $\begin{array}{l}\text { NTP should build on existing partnership with the two } \\
\text { NGOs treating DR-TB to decentralize DR-TB care to } \\
\text { patient's communities. }\end{array}$ \\
\hline & $\begin{array}{l}\text { No transportation available or } \\
\text { challenges to find money to pay for } \\
\text { transportation fares }\end{array}$ & $\begin{array}{l}\text { Immediately Zanmi Lasante (ZL) can use the } 14 \mathrm{MOH} \\
\text { structures supported (in the Central Plateau and } \\
\text { Artibonite and the South), with already existing ZL } \\
\text { staff (trained), stuff and system (network) to provide } \\
\text { DR-TB treatment at district level. GHESKIO can do } \\
\text { the same. }\end{array}$ \\
\hline & $\begin{array}{l}\text { Unreliable referral system from TB } \\
\text { centers to DR-TB facilities }\end{array}$ & $\begin{array}{l}\text { Medium term: collaborate with third parties (WHO, } \\
\text { PAHO) to further extend this decentralization at } \\
\text { district level by putting more resources (training for } \\
\text { staff, equipped lab, drug supply) in the NTP TB } \\
\text { treatments centers throughout the country to also } \\
\text { provide DR-TB treatment. Seek (re-allocate) funds to } \\
\text { provide support necessary to patients during } \\
\text { treatment. }\end{array}$ \\
\hline
\end{tabular}

\section{Funding}

This research was supported by the Abundance foundation, Partners In Health, Haiti / Zanmi Lasante and the Pittsfield Anti-Tuberculosis Association.

\section{Acknowledgements}

The authors express their gratitude to Dr. Paul Farmer, Chair of the Department of Global Health and Social Medicine at Harvard Medical School. The authors thank Staff at Hôpital Bon Sauveur in Cange and Hôpital Sainte Therese in Hinche, and Zanmi Lasante Program direction for their support. The authors express their deepest gratitude to all study participants.

\section{Competing Interests Statement}

The authors declare that they have no competing interests.

\section{References}

Atkinson, P., Coffey, A., Delamont, S., Lofland, J., \& Lofland, L. (2001). Handbook of Ethnography. Thousand Oaks, CA: SAGE Publication. https://doi.org/10.4135/9781848608337

Banerjee, A., Harries, A. D., Nyirenda, T., \& Salaniponi, F. M. (2000). Local perceptions of tuberculosis in a rural 
district in Malawi. International Journal of Tuberculosis and Lung Disease, 4(11), 1047-1051.

Barker, R. D., Millard, F. J. C., Malatsi, J., Mkoana, L., Ngoatwana, T., Agarawal, S., \& De Valliere, S. (2006). Traditional healers, treatment delay, performance status and death from TB in rural South Africa. International Journal of Tuberculosis and Lung Disease, 10(6), 670-675.

Berhanu, R., Schnippel, K., Mohr, E., Hirasen, K., Evans, D., Rosen, S., \& Sanne, I. (2016). Early outcomes of decentralized care for rifampicin-resistant tuberculosis in Johannesburg, South Africa: An observational cohort study. PLoS ONE, 11(11), 1-14. https://doi.org/10.1371/journal.pone.0164974

Corbin, J., \& Strauss, A. (2008). Basics of Qualitative Research: Techniques and Procedures for Developing Grounded Theory. Thousand Oaks, CA: Sage Publications. https://doi.org/10.4135/9781452230153

Cox, H.S., Daniels. J.F., Muller. O., Nicol, M.P., Cox, V., Cutsem, G.V., ... Hughes, J. (2015). Impact of Decentralized Care and the Xpert MTB/RIF Test on Rifampicin-Resistant Tuberculosis Treatment Initiation in Khayelitsha, South Africa. Open Forum Infect Dis, 2(1). https://doi.org/10.1093/ofid/ofv014

Creswell, J. W. (2014). Research design: qualitative, quantitative, and mixed methods approaches (4th ed.). United States: SAGE Publication.

Dharmadhikari, A.S., Mphahlele, M., Venter, K., Stoltz, A., Mathebula, R., Masotla, T., ... Nardell, E. (2014). Rapid impact of effective treatment on transmission of multidrug-resistant tuberculosis. International Journal of Tuberculosis and Lung Disease, 18(9), 1019-1025. https://doi.org/10.5588/ijtld.13.0834

Diel, R., \& Niemann, S. (2013). Outcome of tuberculosis treatment in Hamburg: a survey, 1997-2001. International Journal of Tuberculosis and Lung Diseases, 7(2), 124-31.

Djibuti, M., Mirvelashvili, E., Makharashvili, N., \& Magee, M. J. (2014). Household income and poor treatment outcome among patients with tuberculosis in Georgia: A cohort study. BMC Public Health, 14(1), 1-7. https://doi.org/10.1186/1471-2458-14-88

Farmer, P. (1997). Social scientists and the new tuberculosis. Social Sciences \& Medicine, 44(3), $347-358$. https://doi.org/10.1016/s0277-9536(96)00143-8

Feachem, R., Kjellstrom, T., Murray, C., Over, M., \& Phillips, M. A. (1993). The Health of Adults in the Developing World. New York, NY: Oxford University Press.

Fitzpatrick, C., \& Floyd, K. (2012). A systematic review of the cost and cost effectiveness of treatment for multidrug-resistant tuberculosis. PharmacoEconomics. https://doi.org/10.2165/11595340-000000000-00000

García-García, M., Ponce-de-León, A., García-Sancho, M. C., Ferreyra-Reyes, L., Palacios-Martínez, M., Fuentes, J., ... Sifuentes-Osornio, J. (2002). Tuberculosis-related deaths within a well-functioning DOTS control program. Emerging Infectious Diseases, 8(11), 1327-1333. https://doi.org/10.3201/eid0811.020021

Herrero, M. B., Ramos, S., \& Arrossi, S. (2015). Determinantes da não adesão ao tratamento da tuberculose na. argentina: Barreiras relacionadas com o acesso ao tratamento. Revista Brasileira de Epidemiologia, 18(2), 287-298. https://doi.org/10.1590/1980-5497201500020001

Institute of Medicine. (2011). The New Profile of Drug-Resistant Tuberculosis in Russia: A Global and Local Perspective: Summary of a Joint Workshop by the Institute of Medicine and The Russian Academy of Medical Science. Washington, DC: The National Academies Press. https://doi.org/10.17226/13033

Iseman, M. D., Cohn, D. L., \& Sbarbaro, J. A. (1993). Directly observed treatment of tuberculosis - we can't afford not to try it. New England Journal of Medicine, 328(8), 576-578. https://doi.org/10.1056/NEJM199302253280811

Jacobson, K. R., Theron, D., Kendall, E. A., Franke, M. F., Barnard, M., Van Helden, P. D., ... Warren, R. M. (2013). Implementation of genotype MTBDR plus reduces time to multidrug-resistant tuberculosis therapy initiation in South Africa. Clinical Infectious Diseases, 56(4), 503-508. https://doi.org/10.1093/cid/cis920

Jha, U. M., Satyanarayana, S., Dewan, P. K., Chadha, S., Wares, F., Sahu, S., .. Chauhan, L. S. (2010). Risk factors for treatment default among re-treatment tuberculosis patients in India, 2006. PLoS ONE, 5(1), 2-8. https://doi.org/10.1371/journal.pone.0008873

Kemp, J. R., Mann, G., Simwaka, B. N., Salaniponi, F. M. L., \& Squire, S. B. (2007). Can Malawi's poor afford free tuberculosis services? Patient and household costs associated with a tuberculosis diagnosis in Lilongwe. Bulletin of the World Health Organization, 85(8), 580-585. https://doi.org/10.2471/BLT.06.033167

Loveday, M., Wallengren, K., Brust, J., Roberts, J., Voce, A., Margot, B., ... Padayatchi, N. (2015). 
Community-based care vs. centralised hospitalisation for MDRTB patients, KwaZulu-Natal, South Africa. International Journal of Tuberculosis and Lung Disease, 19(2), 163-171. https://doi.org/10.5588/ijtld.14.0369

Li, Y., Ehiri, J., Tang, S., Li, D., Bian, Y., Lin, H., Marshal, C., \& Cao, J. (2013). Factors associated with patient, and diagnostic delays in Chinese TB patients: A systematic review and meta-analysis. BMC Medicine, $11(156)$.

Mishra, P., Hansen, E. H., Sabroe, S., \& Kafle, K. K. (2005). Socio-economic status and adherence to tuberculosis treatment: A case-control study in a district of Nepal. International Journal of Tuberculosis and Lung Disease, 9(10), 1134-1139.

Morris, M. D., Quezada, L., Bhat, P., Moser, K., Smith, J., Perez, H., ... Rodwell, T. C. (2013). Social, economic, and psychological impacts of MDR-TB treatment in Tijuana, Mexico: A patient's perspective. International Journal of Tuberculosis and Lung Disease, 17(7), 954-960. https://doi.org/10.5588/ijtld.12.0480

Naidoo, P., Du Toit, E., Dunbar, R., Lombard, C., Caldwell, J., Detjen, A., ... Beyers, N. (2014). A comparison of multidrug-resistant tuberculosis treatment commencement times in MDRTBPlus line probe assay and Xpert $\mathbb{R}$ MTB/RIF-based algorithms in a routine operational setting in Cape Town. PLoS ONE, 9(7). https://doi.org/10.1371/journal.pone.0103328

Orsini-Duse, A. G. M/XDR-TB AND THE LAW: South African Context [PowerPoint slides]. Retrieved from https://www.who.int/tb/events/archive/icmeeting_oct07/03_duse.pdf

Patton, M. Q. (2014). Qualitative Research and Evaluation Methods: Integrating Theory and Practice (4th ed.). Los Angeles, CA: SAGE Publication.

Petros, S. G. (2012). Use of a mixed methods approach to investigate the support needs of older caregivers to family members affected by HIV and IADS in South Africa. Journal of Mixed Methods Research, 6(275). https://doi.org/10.1177/1558689811425915

Rouzier, V. A., Oxlade, O., Verduga, R., Gresely, L., \& Menzies, D. (2010). Patient and family costs associated with tuberculosis, including multidrug-resistant tuberculosis, in Ecuador. International Journal of Tuberculosis and Lung Disease, 14(10), 1316-1322.

Rutherford, M. E., Hill, P. C., Maharani, W., Sampurno, H., \& Ruslami, R. (2013). Risk factors for treatment default among adult tuberculosis patients in Indonesia. International Journal of Tuberculosis and Lung Disease, 17(10), 1304-1309. https://doi.org/10.5588/ijtld.13.0084

Verhoogen, E. (1996). The U.S.-Haiti Connection Rich Companies, Poor Workers. Multinational Monitor, 17(4). Retrieved May 19, 2021, from https://www.multinationalmonitor.org/hyper/mm0496.04.html

White, V. L. C., \& Moore-Gillon, J. (1998). Resource implications of patients with multi-drug resistant uberculosis. Thorax, 53(11), 962-963. https://doi.org/10.1136/thorax.55.11.962

Wingfield, T., Tovar, M. A., Huff, D., Boccia, D., Saunders, M. J., Datta, S., ... Evans, C. (2016). Beyond pills and tests: addressing the social determinants of tuberculosis. Clinical Medicine (London, England), 16(6), s79-s91. https://doi.org/10.7861/clinmedicine.16-6-s79

World Health Organization (WHO). (2017a). Global Tuberculosis Report. Retrieved from http://www.who.int/tb/publications/global_report/en/

World Health Organization (WHO). (2017b). Haiti Tuberculosis Profile Report 2017. Retrieved from https://extranet.who.int/sree/Reports?op=Replet\&name=\%2FWHO_HQ_Reports\%2FG2\%2FPROD\%2FE XT\%2FTBCountryProfile\&ISO2=HT\&LAN=EN\&outtype $=\mathrm{html}$

\section{Notes}

Note 1. Name changed to protect patient privacy.

Note 2. We use the term MDR-TB to refer to both forms of resistant TB, as they require the same management approach.

Note 3. BMI normal value; $18.50-24.99$.

Note 4. Presence of new lesion(s) or any worsening of previous lesion(s) is considered abnormal.

Note 5 . $\mathrm{Hb}$ normal value $\geq 12$ for women and $\geq 13$ for men. 
Note 6. Normal range

- $\quad$ Body Mass Index: 18.50- 24.99.

- $\quad$ Hemoglobin: $>12$ in female and $>13$ in male

- $\quad$ Side effects: count (range)

- Depression: $<13$.

- $\quad$ Activities of daily life:0, the higher the number the more restricted the ADL

- Food insecurity: $0=$ little to no hunger in the household, $1=$ moderate to severe hunger in the household

\section{Copyrights}

Copyright for this article is retained by the author(s), with first publication rights granted to the journal.

This is an open-access article distributed under the terms and conditions of the Creative Commons Attribution license (http://creativecommons.org/licenses/by/4.0/). 